\title{
BMJ Open Protocol for developing a Database of Zoonotic disease Research in India (DoZooRI)
}

\author{
Pranab Chatterjee, ${ }^{1,2}$ Soumyadeep Bhaumik, ${ }^{1}$ Abhimanyu Singh Chauhan, ${ }^{1}$ \\ Manish Kakkar ${ }^{1}$
}

To cite: Chatterjee $P$, Bhaumik S, Chauhan AS, et al. Protocol for developing a Database of Zoonotic disease Research in India (DoZooRI). BMJ Open 2017;7:e017825. doi:10.1136/ bmjopen-2017-017825

- Prepublication history and additional material for this paper are available online. To view these files, please visit the journal online (http://dx.doi. org/10.1136/bmjopen-2017017825).

$\mathrm{PC}$ and SB contributed equally.

Received 22 May 2017

Revised 22 September 2017

Accepted 3 November 2017

CrossMark

${ }^{1}$ Public Health Foundation of India, New Delhi, Delhi, India ${ }^{2}$ Division of Epidemiology, National Institute of Cholera and Enteric Diseases, Indian Council of Medical Research, New Delhi, Delhi, India

Correspondence to

Dr Manish Kakkar;

manish.kakkar@phfi.org

\section{ABSTRACT}

Introduction Zoonotic and emerging infectious diseases (EIDs) represent a public health threat that has been acknowledged only recently although they have been on the rise for the past several decades. On an average, every year since the Second World War, one pathogen has emerged or re-emerged on a global scale. Low/middleincome countries such as India bear a significant burden of zoonotic and EIDs. We propose that the creation of a database of published, peer-reviewed research will open up avenues for evidence-based policymaking for targeted prevention and control of zoonoses.

Methods and analysis A large-scale systematic mapping of the published peer-reviewed research conducted in India will be undertaken. All published research will be included in the database, without any prejudice for quality screening, to broaden the scope of included studies. Structured search strategies will be developed for priority zoonotic diseases (leptospirosis, rabies, anthrax, brucellosis, cysticercosis, salmonellosis, bovine tuberculosis, Japanese encephalitis and rickettsial infections), and multiple databases will be searched for studies conducted in India. The database will be managed and hosted on a cloud-based platform called Rayyan. Individual studies will be tagged based on key preidentified parameters (disease, study design, study type, location, randomisation status and interventions, host involvement and others, as applicable).

Ethics and dissemination The database will incorporate already published studies, obviating the need for additional ethical clearances. The database will be made available online, and in collaboration with multisectoral teams, domains of enquiries will be identified and subsequent research questions will be raised. The database will be queried for these and resulting evidence will be analysed and published in peer-reviewed journals.

\section{INTRODUCTION}

Zoonoses, diseases and infections that are naturally transmitted between humans and other vertebrate animals, have been recognised since the beginning of history, but their public health importance has gained increased visibility in the last few decades. ${ }^{1}$ Global analyses of emerging infectious diseases (EIDs) between 1940 and 2004

\section{Strengths and limitations of this study}

The strength of this approach is that in the absence of rigorous surveillance programme and methods to track zoonotic diseases, this approach provides an alternate method to develop a continuously updated database to query zoonotic diseases and their determinants.

- The main limitation of the approach is that it is largely dependent on published data, which may be of heterogenous quality, and could also result in duplicate entries. However, we envisage that the issue of publication bias might be resolved to a large extent in future iterations of the database as collaborators would contribute by adding more citations from the literature to make it a more comprehensive repository of zoonotic disease research in India.

identified that $60.3 \%$ of them were zoonotic and were increasing rapidly over time. ${ }^{2} \mathrm{~A}$ substantial portion of the global burden of endemic zoonoses is from India which is one of the several 'EID hotspots'. ${ }^{34}$ India is experiencing rapid urbanisation, industrialisation, increasing incomes and changing food preferences raising demands for animal source proteins, leading to a larger section of the workforce being in close contact with livestock, including poultry, making a wider section of the population extremely susceptible to zoonotic diseases. Owing to the public health importance of zoonotic diseases in India, a National Standing Committee on Zoonoses was formed in $2007 .{ }^{56}$ Lately, a Centre for Zoonoses Research has been proposed to be set up to harness intersectoral collaborative research using the One Health approach. ${ }^{7}$ However, not much is known about the status of zoonotic disease research and several key aspects related to it, including several aspects of clinical presentation, diagnoses, management and epidemiology in the Indian context. ${ }^{8}$ There is also a need to synthesise evidence about zoonoses in India 
to guide further research, set research and public health priorities and streamline disease control efforts in an evidence-informed manner. ${ }^{910}$ Further, given the resource restrictions and multiple competing priorities that need to be addressed by policymakers and funders alike, the synthesised evidence could help in making evidence-informed decisions to guide resource allocation. This paper reports the protocol for development of a Database of Zoonotic Disease Research in India (DoZooRI), which intends to address these gaps.

\section{Rationale for development of DoZooRI}

We envision DoZooRI as a multidisciplinary research database which would embed all zoonoses-related research in India. DoZooRI will be made accessible to clinicians, veterinarians, researchers, public health professionals, programme managers and policymakers on request. Citations within the research database would be tagged on several carefully chosen key parameters that would enable rapid retrieval of relevant research from a wide variety of sources. This would provide critical support for clinical or public health practice, including, but not limited to the fields of human, veterinary and environmental health, by facilitating evidence syntheses or planning of future research in line with research gaps. The multidisciplinary nature of the database, in fact, provides an impetus to this purpose. This is essential in the context of India, where access to electronic databases as well as trained health information specialists with the capability to develop search strategies is severely limited. ${ }^{11}$ DoZooRI will also address concerns about wasteful research, owing to new research not being based on pre-existing evidence and without their need being vindicated by existing systematic reviews demonstrating critical knowledge gaps. ${ }^{12}{ }^{13}$ Mapping zoonotic disease research and conduct of further evidence syntheses through the DoZooRI will facilitate better understanding of knowledge gaps, which is critical for disease control efforts, policymaking, public health planning and informing public health and research priorities. Further, the database will enable users to analyse research capacity across institutions in India.

DoZooRI is being developed as a strategic tool to support various activities of the India Research Initiative on Peri-Urban Human-Animal-Environment Interface, which is housed at the Public Health Foundation of India, and aims to conduct and support multidisciplinary research, build workforce capacity across sectors and conduct evidence syntheses and policy advocacy.

\section{Implications and possible collaborations}

The creation of the DoZooRI presents an innovative approach to systematic evidence synthesis. It should encourage researchers to collaborate, help to identify and dissolve the compartments which have restricted research efforts to sectoral silos and encourage truly transdisciplinary collaborations to flourish. ${ }^{14-16}$ Using the database, researchers can map zoonotic diseases research; describe the characteristics of interventional research on zoonotic diseases in India; describe the epidemiology of zoonotic diseases, including differences by location, geographic terrain or other determinants; identify the research-policy disconnect and inform on realignment of research priorities in zoonotic disease prevention and control in India; understand institutional capacity to conduct zoonotic research in India and address any other issues, utilising the extended tagging system. The database could potentially be developed into a dynamic repository of the body of work being done in India with respect to zoonotic diseases. Further, if collaborations with techno-medical entrepreneurs can be leveraged, this can be developed into a platform which is updated in real time and allows auto-tagging of published research for the purpose of evidence syntheses. Additionally, this protocol for the development of the database should be of interest to professionals involved with human, animal and environmental health alike, since it outlines the development of a database that proposes curating the first of its kind of evidence base for zoonotic diseases in India. This is likely to be of interest to Indian and international scholars alike, given the global health security threat posed by zoonotic and emerging diseases.

The database is going to provide a step towards closing the evidence-policy gap in zoonotic disease prevention and control programmes by providing the policymakers, programme managers or any other stakeholders access to a searchable, tagged database of existing evidence. This opens avenues for data mining and conducting multiple rapid reviews or systematic reviews to assess the state of the published evidence. The process will bring together the tenets of open science and evidence-based policymaking to strengthen the clinical, veterinarian and public health response to zoonotic disease prevention and control in India.

\section{Objective}

To develop a database of publications resulting from research conducted on a set of priority zoonotic diseases in India and tag them in a manner so as to facilitate further evidence syntheses and landscaping of zoonotic disease research in India.

\section{METHODS AND ANALYSES}

A large-scale systematic mapping of important zoonotic disease research in India will be conducted. Systematic mapping covers a broad cross-section of literature without a narrowly defined research question, as is done in a systematic review and is ideal for the research objective. ${ }^{17-21}$ No quality assessment is done unlike a systematic review but all research on zoonotic diseases is described based on preset characteristics as well as other characteristics identified iteratively as the mapping progresses. The database can, however, serve as a tool for conducting systematic reviews and rapid reviews on focussed research questions on zoonotic disease in India. 
Criteria for inclusion of studies in the database

Definition of zoonotic diseases for the database

We will include research on the following nine priority zoonotic diseases from India, as defined by the study authors ${ }^{22}$ :

1. Leptospirosis

2. Rabies

3. Anthrax

4. Brucellosis

5. Cysticercosis

6. Salmonellosis

7. Bovine tuberculosis

8. Japanese encephalitis

9. Rickettsia infections

We will not include research which has been conducted on participants of Indian origin living abroad or on those who have travelled from India to foreign countries.

Multicentric studies containing Indian participants or samples and systematic reviews including at least one study which meets the population criteria, will be included in the database. For studies conducted before 1947 and where it is not possible to differentiate it with results from post-1947 boundaries by reading the full text of the articles, the boundaries of the undivided British India will be considered.

\section{Study design}

We will include research using any kind of study design except commentaries, editorials, news item, historical articles, meeting notes and narrative reviews. This includes, but is not limited to, animal studies, observational studies, experimental studies, case reports or case series, outbreak investigation, systematic reviews, practice guidelines and qualitative research.

Time limits

We will include research articles irrespective of their year of publication.

\section{Language restriction}

We will include only articles published in English in the database. Exclusion of non-English studies introduces a very small risk of publication bias since English is the dominant language for health research in India.

\section{Publication status}

We will include all articles irrespective of publication status.

\section{Search strategy for electronic databases}

Since the database intends to identify all zoonotic disease research in India, a sensitive search strategy was designed in PubMed using relevant free-text keywords and Medical Subject Heading (MeSH) heading (online supplementary appendix 1). The search strategy was designed iteratively with the intention to achieve maximal sensitivity. This implies that we added additional free-text keywords and $\mathrm{MeSH}$ headings when the search did not yield enough results. Additional free-text keywords were developed by adding synonyms or alternative terms. We then adapted the search strategy for use in other electronic databases, namely CINAHL (EBSCOHost) (online supplementary appendix 2) and Global Health (EBSCOHost) (online supplementary appendix 3). Future versions of DoZooRI might include studies for more electronic databases and additional sources for grey literature. We envision that DoZooRI collaborators who conduct evidence syntheses by using the database will add grey literature citations within the database through expert contact, reference checking of articles from the database, searching clinical trial registries and registers of observational studies and systematic reviews.

\section{Screening of articles}

We will screen all citations retrieved independently by at least two researchers based on titles and abstracts (if available) and mark them as 'retrieve' (eligible or unclear eligibility) or 'do not retrieve' (not eligible). For all citations marked 'retrieve', full texts will be acquired and final decision on eligibility will be taken independently by two researchers. In case of any discrepancies that are not resolved through discussion between the two reviewers (SB and PC), consensus will be achieved by consultation with a third expert researcher (MK). Reasons for exclusion at full-text phase will be documented.

\section{Data management}

We will manage data and host the database in a cloudbased platform called Rayyan (http://rayyan.qcri.org/). The platform allows simultaneous screening as well as tagging of citations in a blinded fashion by multiple researchers. Rayyan also allows multiple tagging of each citation. We chose Rayyan over other options because of its two-fold advantages-it is free to use and it has an inbuilt artificial platform where it provides prediction for includes and excludes based on the choices marked by researchers manually through a colour-coded five-star system by text mining the records. An independent evaluation of Rayyan found that after screening of three-quarters of included citations $98 \%$ of articles included were correctly predicted. ${ }^{23}$ This would be an essential tool to rapidly allow the DoZooRI administrators to screen and mark includes and excludes when new studies are added as and when the database is updated from time to time. Rayyan also detects duplicates automatically.

\section{Database tagging}

We will develop a tagging system iteratively for several key parameters, which would enable users to rapidly identify and retrieve articles to answer research, policy or clinical questions of their interest. Tagging will be done independently by at least two researchers (SB and PC) and discrepancies will be solved through consensus decision within the research team. We have predetermined tags for the following key parameters:

- Name(s) of zoonotic disease-from among the nine priority zoonotic diseases. 
- Study design-classification as per standard terminologies (eg, case report or case series, case-control, cohort, cross-sectional, retrospective study, ecological study, clinical trial, systematic review, qualitative research and in vitro studies).

- Special study type tags-for example, GENE for genetic or genomic studies, EPIDEM for a study on any aspect of disease epidemiology, OUTBREAK for a study on any aspect of disease outbreak, DIAG for a study on diagnostic modality for a zoonotic disease and ECONOM on health economics-related studies.

- For epidemiological studies-name of the state(s) of India where the study was conducted.

- For clinical trials-randomisation status and name of intervention.

- Based on host involved: human, cattle, wild animals, multihost, invertebrate vectors and so on.

\section{ETHICS AND DISSEMINATION}

We prepared DoZooRI from already conducted research publications and did not involve any animal or human subjects. As such, there are no ethical issues involved in the creation of the database or its subsequent use for evidence syntheses.

The findings from any research done by using DoZooRI will be published in peer-reviewed journals. The results of any such research on the database will also be shared with key stakeholders, including health professionals, researchers and policymakers in the form of policy briefs.

Contributors MK and PC conceptualised the article; SB and ASC were responsible for developing the initial methods; PC and SB refined the methodology and pilot tested it using a limited exercise and drew up the first draft of the manuscript. All the authors contributed to the development and drafting of the manuscript, including reviews and intellectual inputs in the process. All the authors have reviewed and approved the final version of the manuscript which has been submitted to the journal.

Funding Funding received from International Development Research Centre Grant No. 107344-001.

Competing interests None declared.

Provenance and peer review Not commissioned; externally peer reviewed.

Open Access This is an Open Access article distributed in accordance with the Creative Commons Attribution Non Commercial (CC BY-NC 4.0) license, which permits others to distribute, remix, adapt, build upon this work non-commercially, and license their derivative works on different terms, provided the original work is properly cited and the use is non-commercial. See: http://creativecommons.org/ licenses/by-nc/4.0/ (c) Article author(s) (or their employer(s) unless otherwise stated in the text of the article) 2017. All rights reserved. No commercial use is permitted unless otherwise expressly granted.

\section{REFERENCES}

1. Hubálek Z. Emerging human infectious diseases: anthroponoses, zoonoses, and sapronoses. Emerg Infect Dis 2003;9:403-4.

2. Jones KE, Patel NG, Levy MA, et al. Global trends in emerging infectious diseases. Nature 2008;451:990-3.

3. King L. Neglected Zoonotic diseases. In: Institute of Medicine (US), Forum on Microbial Threats, ed. The causes and impacts of neglected tropical and Zoonotic diseases: opportunities for integrated intervention strategies. Washington: National Academies Press, 2011.

4. Woolhouse ME. Epidemiology: emerging diseases go global. Nature 2008;451:898-9.

5. Planning Commission Working Group 3. Report of the working group on disease burden for the 12th five year plan: The Planning Commission, New Delhi, 2010.

6. Ramthanga IC. The Mizoram gazette extra ordinary published by authority. Mizoram Gaz. 2008;37:1-2.

7. Chatterjee P, Kakkar M, Chaturvedi S. Integrating one health in national health policies of developing countries: India's lost opportunities. Infect Dis Poverty 2016;5:87.

8. Sekar N, Shah NK, Abbas SS, et al. Research options for controlling zoonotic disease in India, 2010-2015. PLoS One 2011;6:e17120.

9. Abbas SS, Kakkar M. Research \& policy disconnect: the case of rabies research in India. Indian J Med Res 2013;138:560-1.

10. Abbas SS, Kakkar M. Rabies control in India: a need to close the gap between research and policy. Bull World Health Organ 2015;93:131-2.

11. Chatterjee $P$, Biswas $T$, Datta $A$, et al. Healthcare information and the rural primary care doctor. S Afr Med J 2012;102:138-9.

12. Moher D, Glasziou P, Chalmers I, et al. Increasing value and reducing waste in biomedical research: who's listening? Lancet 2016;387:1573-86.

13. Chalmers I, Glasziou P. Systematic reviews and research waste. Lancet 2016;387:122-3.

14. Max-Neef MA. Foundations of transdisciplinarity. Ecol Econ 2005;53:5-16.

15. Gray B. Enhancing transdisciplinary research through collaborative leadership. Am J Prev Med 2008;35:S124-32.

16. Stokols $\mathrm{D}$. Toward a science of transdisciplinary action research. $A m$ $J$ Community Psychol 2006;38:79-93.

17. Chersich M, Becerril-Montekio V, Becerra-Posada F, et al. Perspectives on the methods of a large systematic mapping of maternal health interventions. Global Health 2016;12:51.

18. Pigott DM, Howes RE, Wiebe A, et al. Prioritising infectious disease mapping. PLoS Negl Trop Dis 2015;9:e0003756.

19. Bezerra F, Favacho CH, Souza R, et al. Towards supporting systematic mappings studies: an automatic snowballing approach. 29th SBBD proceedings. Curitiba, Brazil: SBBD, 2014:167-76

20. Cooper ID. What is a "mapping study?". J Med Libr Assoc 2016;104:76-8.

21. Hay SI, Battle KE, Pigott DM, et al. Global mapping of infectious disease. Philos Trans R Soc Lond B Biol Sci 2013;368:20120250.

22. Public Healh Foundation of India. Roadmap to Combat Zoonoses in India (RCZI) initiative. 2016. http://zoonoses.phfi.org/ (accessed 15 Dec 2016)

23. Olofsson $\mathrm{H}$, Brolund $\mathrm{A}$, Hellberg $\mathrm{C}$, et al. Can abstract screening workload be reduced using text mining? User experiences of the tool Rayyan. Res Synth Methods 2017;8:275-80. 